\title{
THE EXCHANGEABLE POTASSIUM CONTENT IN DISEASE STATES ${ }^{1,2}$
}

\author{
BY JERRY K. AIKAWA, ${ }^{3}$ JOHN H. FELTS, JR., MALCOLM P. TYOR, AND \\ GEORGE T. HARRELL WITH THE TECHNICAL ASSISTANCE OF \\ ELOISE L. RHOADES

\begin{abstract}
(From the Department of Internal Medicine, Bowman Gray School of Medicine of Wake Forest College, and the North Carolina Baptist Hospital, Winston-Salem, N. C.)
\end{abstract}

(Submitted for publication February 2, 1952; accepted May 26, 1952)

Although the importance of potassium in physiologic processes has long been recognized, there is a paucity of information concerning its metabolism, particularly in clinical disease states. This scarcity of knowledge has been due primarily to the technical difficulties involved in measurement of soluble cations. The introduction of flame spectrophotometry and the availability of synthetic radioactive isotopes have partially overcome these technical limitations.

Although information is available concerning the external balance of potassium and alterations in the extracellular potassium concentration in various disease states, such data give no direct information concerning the body store of this cation. The injection of radioactive potassium $\left(\mathrm{K}^{42}\right)$ affords the only direct procedure available for estimating the total potassium content of the body in the intact animal. The specific activity of the urine, after partial equilibration of radioactive potassium with the native atoms, can be used as a measure of the "total exchangeable potassium content" $(\mathrm{Ke})$. Values for normal young males and females have been reported $(1,2)$. No such studies in individuals with various disease states are yet available.

The purpose of the present study was to determine the extent to which pathologic conditions alter the exchangeable potassium content of hospitalized subjects, and to attempt to correlate alterations in this measurement with the associated metabolic disturbances, and to determine if the

1 This work was supported in part by a grant-in-aid from the American Heart Association and in part by the U. S. Atomic Energy Commission through a contract with the Bowman Gray School of Medicine.

2 The $\mathrm{K}^{\mathbf{w}}$ used was supplied by the National Laboratories, Oak Ridge, Tennessee, on allocation from the U. S. Atomic Energy Commission.

3 Research Fellow of the American Heart Association. administration of "loading" doses of potassium could correct deficiencies detected.

\section{MATERIAL AND METHODS}

Subjects

A total of 69 hospitalized subjects-39 males and 30 females-between the ages of 14 and 78 years were studied. The various pathologic states observed are listed in Tables I-IV. All had illnesses of some weeks' duration which justified hospitalization for study and treatment. In addition, six normal individuals, three males and three females, were studied in order to determine the reproducibility of the measurement and the effect of the administration of an oral supplement of potassium.

The subjects were divided into four groups. Group 1 consisted of the normal subjects and the four patients in steady states. Groups 2 and 3, consisting, respectively, of 28 males and 22 females, were used for the original survey study. The individuals in these two groups were selected at random from patients admitted to the medical wards. Studies were performed on any such patient from whom reliable urine collections could be made. Because facilities were limited, it was not possible to include all likely candidates in the study.

Group 4, consisting of nine males and six females, was studied a few months after the study of groups 2 and 3 was completed. In this group serial determinations of $\mathrm{Ke}$ were performed in order to study the effect of supplementary potassium chloride. At least two determinations of $\mathrm{Ke}$ were performed on each patient in this group.

\section{Plan of the experiment}

In the normal subjects in group 1 , two determinations of $\mathrm{Ke}$ were performed one week apart; the subjects were then given $\mathrm{KCl}, 3 \mathrm{gm}$. daily for six days, and the third $\mathrm{Ke}$ was measured. The manner in which the other four were studied is described under Comments in Table I.

Single determinations of $\mathrm{Ke}$ were done in groups 2 and 3. In group 4, the initial determination of $\mathrm{Ke}$ was performed as soon as possible after the patient's admission to the hospital. Upon completion of the initial study, 13 subjects were given oral supplements of potassium chloride, $3 \mathrm{gm}$. (40 meq.) daily, for a week. Then a second Ke determination was done after which potassium chloride was discontinued. Whenever possible a third determination was performed a week later. Two of the individuals in group 4 were not given supplementary potassium chlo- 
ride; in these cases, the effect of the disease process was observed by serial determinations of $\mathrm{Ke}$.

\section{Experimental procedure}

After breakfast, between 8:30 and 9 a.m., each subject was given an intravenous injection of radioactive potassium by calibrated syringe. All urine specimens until 6 a.m. the next morning were collected and pooled, and this collection of urine was measured for the excretion of $\mathrm{K}^{\mathrm{s}}$. The specific activity of the urine was determined on two spot samples collected at 7 and 9 a.m. on the day after injection. The subject was asked to empty his bladder completely by voiding. In some instances, a specimen could not be obtained at the exact time, but was obtained as soon thereafter as possible.

No restrictions were placed on activity or on the intake of food, water, or salt. The usual lunch and supper were given on the day of injection. Breakfast the next morning was delayed until after the second spot urine sample was obtained.

Preparation and administration of radioactive potassium

All shipments of $\mathrm{K}^{\text {so }}$ were treated in the manner described by Corsa and his co-workers (1). Usable ship- ments were usually received early Tuesday morning, and most of the injections were made later that morning. Approximately $1.5 \mathrm{meq}$. of potassium chloride solution, containing 100 microcuries of $\mathrm{K}^{22}$, were injected intravenously. A few injections were given on Wednesday, and these patients received 3 meq. of potassium chloride solution containing 50 microcuries of $\mathrm{K}^{23}$.

\section{Measurement of radioactivity}

The activity of the urine specimens was determined with a dipping tube and a scaling circuit. Counts were made to $1 \%$ accuracy. All eounting rates were at least ten times background, and were usually in the range of 500 to 3,000 per minute. At this range of counting rate, no dead time correction for the Geiger tube was necessary. All determinations were corrected for decay.

Preliminary studies confirmed the observations of Corsa and his associates (1) that the specific activity of potassium in the urine reached an equilibrium by 24 hours. The mean difference in specific activity between the two spot specimens, when expressed as per cent of the mean $\mathrm{Ke}$, was $6.52 \pm 6.65 \%$.

TABLE I

Reproducibility of exchangeable potassium measurements and the effect of oral supplements of potassium in normal subjects and in patients in steady states

\begin{tabular}{|c|c|c|c|c|c|c|}
\hline \multirow{2}{*}{ Subjects } & \multicolumn{2}{|c|}{$\mathrm{Ke}_{\mathbf{1}}$} & \multicolumn{2}{|c|}{ Kez } & \multicolumn{2}{|c|}{ Kes } \\
\hline & (meq.) & (meq./Kg.) & (meq.) & $($ meq./Kg.) & (meq.) & (meq./Kg.) \\
\hline \multicolumn{7}{|c|}{ Normal Subjects } \\
\hline $\begin{array}{c}\text { Women } \\
1 \\
2 \\
3 \\
\text { Men } \\
1 \\
2 \\
3\end{array}$ & $\begin{array}{l}2,500 \\
1,947 \\
2,160 \\
\\
3,641 \\
3,537 \\
4,064\end{array}$ & $\begin{array}{l}45.9 \\
39.3 \\
38.9 \\
\\
43.3 \\
48.1 \\
38.1\end{array}$ & $\begin{array}{l}2,219 \\
1,682 \\
2,155 \\
\\
3,447 \\
3,261 \\
4,007\end{array}$ & $\begin{array}{c}40.7 \\
34.0 \\
38.8 \\
\\
41.0 \\
44.3 \\
37.5\end{array}$ & $\begin{array}{l}2,280 \\
1,679 \\
2,358 \\
\\
4,145 \\
3,753 \\
4,136\end{array}$ & $\begin{array}{l}38.2^{*} \\
33.9^{*} \\
42.5^{*} \\
49.3^{*} \\
51.0^{*} \\
38.7^{*}\end{array}$ \\
\hline
\end{tabular}

Patients in Steady States

\begin{tabular}{|c|c|c|c|c|c|c|c|c|c|}
\hline \multirow{2}{*}{ Patient } & \multirow{2}{*}{$\begin{array}{c}\text { Age, } \\
\text { sex }\end{array}$} & \multirow{2}{*}{ Diagnosis } & \multicolumn{2}{|r|}{$\mathbf{K e}_{\mathbf{1}}$} & \multicolumn{2}{|r|}{$\mathrm{Ke}_{2}$} & \multicolumn{2}{|c|}{$\mathrm{Kez}$} & \multirow{2}{*}{ Comments } \\
\hline & & & (meq.) & (meq./Kg.) & (meq.) & (meq./Kg.) & (meq.) & (meq./Kg.) & \\
\hline $\mathrm{Ba}$ & M 71 & $\begin{array}{l}\text { Diabetes with periph- } \\
\text { eral vascular disease }\end{array}$ & 1,544 & 25.3 & 1,524 & 24.9 & 1,252 & $20.6^{*}$ & $\begin{array}{l}\text { Diabetes well regulated } \\
\text { Supracondylar amputation }\end{array}$ \\
\hline Ho & M 72 & Diabetes with periph- & 1,848 & 34.7 & 1,750 & $32.9^{*}$ & 1,894 & 36.6 & Diabetes well regulated \\
\hline Bo & F 59 & $\begin{array}{l}\text { Myxedema and dia- } \\
\text { betes }\end{array}$ & 1,553 & 21.4 & 1,576 & 21.7 & & & $\begin{array}{l}\text { Myxedema for several years. } \\
\text { Diabetes well regulated. No }\end{array}$ \\
\hline Th & F 38 & Ulcerative colitis & 1,641 & 34.7 & 1,638 & 34.6 & 1,811 & 37.6 & $\begin{array}{l}\text { Duration } 8 \text { years. In relapse } \\
\text { during study. Interval of } \\
2 \text { wks. between } \mathrm{Ke}_{2} \text { and } \mathrm{Ke}_{3}\end{array}$ \\
\hline
\end{tabular}

All Ke determinations performed at weekly intervals unless otherwise noted.

* Ke determination after administration of oral supplement of $\mathrm{KCl}, 3 \mathrm{gm}$. daily, for six days. 
TABLE II

Exchangeable potassium content in males with disease states (group 2)

\begin{tabular}{|c|c|c|c|c|c|c|}
\hline Patient & $\begin{array}{c}\text { Age } \\
(y r s .)\end{array}$ & Diagnosis & $\underset{(m e q .)}{\mathrm{Ke}}$ & $\begin{array}{c}\text { Body } \\
\text { weight } \\
(K g .)\end{array}$ & $\underset{(\text { meq./Kg. })}{\mathrm{Ke} / \mathrm{Wt} .}$ & Remarks \\
\hline 1 & 32 & $\begin{array}{l}\text { Hypertensive cardiovascular } \\
\text { disease }\end{array}$ & 3,797 & 64.5 & 58.9 & $\begin{array}{l}\text { Duration } 11 \text { yrs. Symptomatic for } 6 \text { mos. } \\
\text { Clinically-well nourished. }\end{array}$ \\
\hline 2 & 51 & Neurosyphilis & 4,016 & 68.3 & 58.8 & $\begin{array}{l}\text { Spinal fluid protein }=150 \mathrm{mg} . \% \text {. Clini- } \\
\text { cally well nourished. }\end{array}$ \\
\hline 3 & 48 & $\begin{array}{l}\text { Polycystic kidneys with azo- } \\
\text { temia }\end{array}$ & 3,289 & 60.8 & 54.1 & $\begin{array}{l}\text { Hypertension for } 3 \text { yrs. NPN }=166 \mathrm{mg} \text {. } \\
\% \text {. No generalized edema. }\end{array}$ \\
\hline 4 & 42 & Ulcerative colitis & 2,767 & 53.6 & 51.6 & Duration 6 mos. 34 lbs. weight loss. \\
\hline 5 & 66 & $\begin{array}{l}\text { Pneumoconiosis and pulmo- } \\
\text { nary emphysema }\end{array}$ & 2,772 & 54.1 & 51.2 & Arteriosclerotic heart disease. Slender. \\
\hline 6 & 37 & Pyelonephritis & 3,097 & 61.4 & 50.4 & $\begin{array}{l}\text { Minimal symptoms for } 6 \text { mos. No recent } \\
\text { weight loss or generalized edema. NPN } \\
=65 \mathrm{mg} . \% \text {. }\end{array}$ \\
\hline 7 & 56 & Iron-deficiency anemia & 2,942 & 60.0 & 49.0 & Duration 6 mos. Weight loss of $15 \mathrm{lbs}$. \\
\hline 8 & 49 & $\begin{array}{l}\text { Duodenal ulcer, adenocarci- } \\
\text { noma of cecum }\end{array}$ & 2,608 & 54.5 & 47.9 & $\begin{array}{l}\text { Clinically emaciated. Weight loss of } 20 \\
\text { lbs. in } 5 \text { mos. No anemia or hypopro- } \\
\text { teinemia. }\end{array}$ \\
\hline 9 & 25 & $\begin{array}{l}\text { Convulsive disorder, pyrexia of } \\
\text { undetermined origin }\end{array}$ & 2,679 & 57.5 & 46.6 & $\begin{array}{l}\text { Symptomatic } 2 \text { wks. Afebrile at time of } \\
\text { determination. }\end{array}$ \\
\hline 10 & 71 & Generalized arteriosclerosis & 2,629 & 56.6 & 46.4 & $\begin{array}{l}\text { Chronic blood loss from internal hemor- } \\
\text { rhoids. Hemoglobin }=10.5 \mathrm{gm} \text {. }\end{array}$ \\
\hline 11 & 58 & Diabetes mellitus & 2,088 & 45.8 & 45.6 & $\begin{array}{l}\text { Duration } 3 \text { yrs. Poorly controlled } 8 \text { mos., } \\
\text { with } 20 \text { lbs. weight loss. }\end{array}$ \\
\hline 12 & 19 & Peptic ulcer & 2,912 & 65.1 & 44.7 & $\begin{array}{l}\text { Epigastric symptoms for } 18 \text { mos. Hema- } \\
\text { temesis } 8 \text { days prior to admission. }\end{array}$ \\
\hline 13 & 14 & Congenital heart disease & 1,358 & 30.5 & 44.5 & $\begin{array}{l}\text { Interventricular septal defect. Cryptor- } \\
\text { chidism. }\end{array}$ \\
\hline 14 & 16 & Trichinosis, peptic ulcer & 2,478 & 55.9 & 44.3 & $\begin{array}{l}\text { Duration } 2.5 \text { wks. Eosinophilia }(25 \%) \text {. } \\
\text { Positive trichinella skin test. }\end{array}$ \\
\hline 15 & 40 & Cerebral aneurysm & 3,282 & 79.1 & 41.5 & $\begin{array}{l}\text { Symptoms limited to complaint of head- } \\
\text { aches. Normal otherwise. }\end{array}$ \\
\hline 16 & 56 & Neur & 2,370 & 57.7 & 41.1 & Spinal fluid protein $=102 \mathrm{mg} . \%$ \\
\hline 17 & 49 & Diak & 3,3 & 86.8 & 0 & $\begin{array}{l}\text { Well regulated. Admitted for therapy of } \\
\text { acute upper respiratory infection. }\end{array}$ \\
\hline 18 & 58 & $\begin{array}{l}\text { Chronic cholecystitis with chol- } \\
\text { elithiasis }\end{array}$ & 2,801 & 73.0 & 38.4 & Faint icterus. Clinically well nourished. \\
\hline 19 & 56 & $\begin{array}{l}\text { Rheumatic and arteriosclerotic } \\
\text { heart disease }\end{array}$ & 2,535 & 69.3 & 36.6 & $\begin{array}{l}\text { Digitalized; no edema or weight loss. Auric- } \\
\text { ular fibrillation. }\end{array}$ \\
\hline 20 & 41 & Progressive peroneal atrophy & 1, & 43.4 & 36.0 & Duration 30 yrs. \\
\hline 21 & 57 & Port: & 2, & 61.3 & 33.5 & $\begin{array}{l}\text { Neurodermatitis disseminata for } 8 \text { mos. } 28 \\
\text { lbs. weight loss in } 2 \text { mos. } 45 \% \text { BSP reten- } \\
\text { tion. }\end{array}$ \\
\hline 22 & 76 & Arteriosclerotic heart disease & 2,500 & 75.2 & 33.3 & $\begin{array}{l}\text { Progressive congestive heart failure } 14 \text { mos. } \\
20 \text { lbs. weight loss in } 1 \text { yr. Pitting edema. }\end{array}$ \\
\hline 23 & 78 & $\begin{array}{l}\text { Adenocarcinoma of head of } \\
\text { pancreas }\end{array}$ & 1,329 & 42.3 & 31.4 & $\begin{array}{l}30 \text { lbs. weight loss in } 1 \text { mo. prior to admis- } \\
\text { sion. Known weight loss for } 1 \text { yr. Ano- } \\
\text { rexic. }\end{array}$ \\
\hline 24 & 69 & $\begin{array}{l}\text { Thyrotoxicosis, arteriosclerotic } \\
\text { heart disease }\end{array}$ & 1,413 & 45.5 & 31.1 & $\begin{array}{l}\text { Parkinsonism } 35 \text { yrs. Duration of thyro- } \\
\text { toxicosis, } 8 \text { mos. In relapse. }\end{array}$ \\
\hline 25 & 56 & $\begin{array}{l}\text { Arteriosclerotic heart disease, } \\
\text { pulmonary embolism }\end{array}$ & 1,437 & 48.0 & 29.9 & $\begin{array}{l}\text { Duration heart disease } 15 \text { mos. with } 40 \text { lbs. } \\
\text { weight loss. Loss of } 20 \mathrm{lbs} \text {. in } 10 \text { days on } \\
\text { mercurials and digitalis. }\end{array}$ \\
\hline 26 & 42 & Metastatic carcinoma & 2,350 & 82.7 & 28.4 & $\begin{array}{l}\text { Weight loss } 20 \text { lbs. in preceding } 4 \text { mos. Per- } \\
\text { sistent fever. Primary site in colon. }\end{array}$ \\
\hline 27 & 55 & $\begin{array}{l}\text { Malnutrition, empyema, pneu- } \\
\text { mothorax }\end{array}$ & 864 & 32.5 & 26.6 & $\begin{array}{l}\text { Hypertensive cardiovascular disease. } 75 \\
\text { lbs. weight loss. Expired } 10 \text { days after } \\
\text { determination. }\end{array}$ \\
\hline 28 & 16 & $\begin{array}{l}\text { Inactive rheumatic heart dis- } \\
\text { ease }\end{array}$ & 1,667 & 67.7 & 24.6 & $\begin{array}{l}\text { Acute rheumatic fever } 3 \text { yrs. previously. } \\
\text { Normal sedimentation rate at time of de- } \\
\text { termination. }\end{array}$ \\
\hline
\end{tabular}


Chemical determinations

Total potassium concentration in the urine was determined by flame spectrophotometry, using the method of Mosher and his associates (3).

\section{Calculation of exchangeable potassium}

The following formula was used to calculate the value for the exchangeable potassium content of the body:

$$
\mathrm{Ke}=\frac{\mathrm{K}_{\mathrm{l}}^{42}-\mathrm{K}_{\mathrm{o}}^{42}}{\frac{\mathrm{Ku}^{42}}{\mathrm{Ku}^{39}}} .
$$

$\mathrm{Ke}=$ quantity of exchangeable potassium in milliequivalents.

$\mathrm{K}_{\mathrm{l}}{ }^{42}=$ quantity of radiopotassium administered (arbitrary units).

$\mathrm{K}_{\mathrm{o}}{ }^{42}=$ quantity of radiopotassium excreted in the urine within 21 to 21.5 hours after its intravenous injection.

$\mathrm{Ku}^{42}=$ concentration of radiopotassium in the urine samples.

$\mathrm{Ku}^{42} / \mathrm{Ku}^{39}=$ mean specific activity of two spot specimens.
RESULTS

\section{Group 1 (Table I)}

Better reproducibility of $\mathrm{Ke}$ was obtained in patients in steady states than in normal young subjects. However, the activity and the diet of the individuals during the study was better controlled in the patients. The mean difference between the two $\mathrm{Ke}$ determinations made a week apart without oral supplementation was 125 meq. or 2.0 meq. $/ \mathrm{Kg}$. Following the administration of an oral supplement, the second $\mathrm{Ke}$ was higher by a mean of 212 meq. or 2.2 meq./Kg. In the interpretation of the results in the other groups, an increase of greater than 250 meq. or 5 meq. $/ \mathrm{Kg}$. was considered of significance, although it is rec-

TABLE III

Exchangeable potassium content in females with disease states (group 3)

\begin{tabular}{|c|c|c|c|c|c|c|}
\hline Patient & $\begin{array}{c}\text { Age } \\
(y r s .)\end{array}$ & Diagnosis & $\underset{(m e q .)}{\mathrm{Ke}}$ & $\begin{array}{l}\text { Body } \\
\text { weight } \\
(K g .)\end{array}$ & $\underset{(m e q . / K g .)}{\mathrm{Ke} / \mathrm{Wt} .}$ & Remarks \\
\hline 1 & 25 & Diabetes mellitus & 2,201 & 43.6 & 50.5 & $\begin{array}{l}\text { Duration } 9 \text { yrs. Poor control. Urinary } \\
\text { sugar } 3+\text { on day of determination. }\end{array}$ \\
\hline 2 & 55 & Diabetes mellitus & 2,540 & 55.9 & 45.5 & $\begin{array}{l}\text { Clinically well nourished. Duration } 4 \text { yrs. } \\
\text { with poor control. Neurogenic bladder. }\end{array}$ \\
\hline 3 & 34 & Chronic anxiety state & 1,811 & 45.2 & 40.1 & Duration 2 yrs. Weight loss 20 lbs. \\
\hline 4 & 54 & Vascular lesion, basal ganglion & 1,805 & 46.4 & 38.9 & $\begin{array}{l}\text { Tremor of right arm, progressive for } 3 \mathrm{yrs.} \\
\text { Recent involvement of right leg. }\end{array}$ \\
\hline 5 & 67 & Gastric ulcer & 1,368 & 36.0 & 38.0 & Very slender. Quiescent bronchial asthma. \\
\hline 6 & 42 & $\begin{array}{l}\text { Hypertensive cardiovascular } \\
\text { disease }\end{array}$ & 1,789 & 47.5 & 37.7 & No history of weight loss. \\
\hline 7 & 59 & $\begin{array}{l}\text { Hypertensive and arterioscle- } \\
\text { rotic heart disease }\end{array}$ & 2,230 & 60.9 & 36.6 & $\begin{array}{l}\text { Pulmonary fibrosis. Digitoxin, } 0.8 \mathrm{mg} . \text {, } \\
\text { given during determination. }\end{array}$ \\
\hline 8 & 52 & Psychoneurosis & 1,498 & 43.2 & 34.7 & $\begin{array}{l}\text { Weight loss and diarrhea for several months. } \\
\text { Narcotic addiction. }\end{array}$ \\
\hline 9 & 21 & $\begin{array}{l}\text { Ulcerative colitis, emetine in- } \\
\text { toxication }\end{array}$ & 1,551 & 44.8 & 34.6 & $\begin{array}{l}\text { Duration colitis, } 7 \text { yrs. Emetine therapy } \\
1 \text { mo. previously; subsequently developed } \\
\text { generalized weakness and tachycardia. }\end{array}$ \\
\hline 10 & 43 & Duodenitis, anemia & 1,666 & 51.8 & 32.2 & $\begin{array}{l}\text { Iron-deficiency anemia. Asymptomatic in- } \\
\text { testinal amebiasis. }\end{array}$ \\
\hline 11 & 41 & Rheumatic heart disease & 1,726 & 54.1 & 31.9 & $\begin{array}{l}\text { Mitral stenosis and insufficiency. On digi- } \\
\text { talis and quinidine. }\end{array}$ \\
\hline 12 & 54 & Rheumatoid arthritis & 1,609 & 55.9 & 28.8 & $\begin{array}{l}\text { Progressive disease for } 2 \text { yrs. Bronchial } \\
\text { asthma for } 30 \text { yrs. }\end{array}$ \\
\hline $\begin{array}{l}13 \\
14\end{array}$ & $\begin{array}{l}30 \\
55\end{array}$ & Exogenous obesity & 2,700 & 95.2 & 28.4 & Typical fat distribution. \\
\hline 15 & $\begin{array}{l}30 \\
21\end{array}$ & Chronic anxiety state & $\begin{array}{l}2,031 \\
1,439\end{array}$ & 50.9 & 28.3 & $\begin{array}{l}\text { Emotional immaturity. Clinically simu- } \\
\text { lated thyrotoxicosis. Weight loss of } 7 \mathrm{lbs} \text {. } \\
\text { in } 3 \mathrm{wks} \text {. before admission. }\end{array}$ \\
\hline 16 & 24 & Multiple sclerosis & 1,380 & 49.5 & 27.9 & $\begin{array}{l}\text { In relapse. Duration of illness, } 1 \mathrm{yr} \text {. No } \\
\text { recent weight loss. }\end{array}$ \\
\hline 17 & 65 & Generalized urticaria & 1,435 & 53.2 & 27.0 & $\begin{array}{l}\text { Urticaria for } 2.5 \text { mos., etiology unknown. } \\
\text { Postmenopausal osteoporosis. }\end{array}$ \\
\hline 18 & 73 & $\begin{array}{l}\text { Rheumatoid arthritis and cere- } \\
\text { brovascular accident }\end{array}$ & 1,221 & 47.3 & 25.8 & $\begin{array}{l}\text { Chronic disease state. Arthritis for several } \\
\text { years. Generalized arteriosclerosis. }\end{array}$ \\
\hline 19 & 44 & Obesity, anemia & 1,675 & 67.5 & 24.8 & $\begin{array}{l}\text { Iron-deficiency type. Anticoagulant ther- } \\
\text { apy for retinal vein thrombosis. }\end{array}$ \\
\hline \multirow{3}{*}{$\begin{array}{l}20 \\
21\end{array}$} & 19 & Diabetes mellitus & 1,673 & 72.0 & 23.2 & Duration 2 yrs. Urinary sugar $4+$. \\
\hline & 44 & Psychoneurosis & 1,307 & 64.5 & 20.3 & $\begin{array}{l}\text { Tension headaches, psychic anorexia, nausea } \\
\text { and vomiting. Weight loss of } 30 \mathrm{lbs} \text {. }\end{array}$ \\
\hline & 45 & $\begin{array}{l}\text { Exogenous obesity, hyperten- } \\
\text { sive cardiovascular disease }\end{array}$ & 1,281 & 68.2 & 18.8 & Mild cerebrovascular accident. \\
\hline
\end{tabular}


ognized that the retention of administered potassium over a short period may not be definite evidence for a prior depletion.

\section{Group 2 (Table II)}

When the values for $\mathrm{Ke} / \mathrm{Wt}$. from this group of male subjects with various disease states were compared with those found in 30 normal young males (in whom the range was $35.6-53.6$ meq./ $\mathrm{Kg}$.), three subjects (Cases 1, 2, and 3) had values higher than those found in the normal males. Eight of the 28 subjects had values lower than $35.6 \mathrm{meq} . / \mathrm{Kg}$. The disease states in which these low values were found included inactive rheumatic

TABLE IV

Exchangeable potassium content in subjects studied serially (group 4)

\begin{tabular}{|c|c|c|c|c|c|c|}
\hline $\begin{array}{l}\text { Patient. } \\
\text { sex }\end{array}$ & $\begin{array}{c}\text { Age } \\
(y r s .)\end{array}$ & Diagnosis & $\underset{(m e q .)}{\mathrm{Ke}}$ & $\begin{array}{l}\text { Body } \\
\text { weight } \\
(K g .)\end{array}$ & $\begin{array}{c}\mathrm{Ke} / \mathrm{Wt} \\
(m e q . / K g .)\end{array}$ & Remarks \\
\hline roup & & & & & & \\
\hline $1 \mathrm{M}$ & 39 & $\begin{array}{l}\text { Carcinoma metastatic to } \\
\text { the liver }\end{array}$ & $\begin{array}{l}1,566^{*} \\
1,600 \dagger\end{array}$ & $\begin{array}{l}55.0 \\
51.0\end{array}$ & $\begin{array}{l}28.5 \\
31.4\end{array}$ & $\begin{array}{l}\text { Onset with weakness and weight loss of } 20 \text { lbs. } \\
\text { in } 5 \text { mos. Confirmed by liver biopsy. }\end{array}$ \\
\hline $2 \mathrm{~F}$ & 65 & Myxedema & $\begin{array}{l}1,586^{*} \\
1,366 \dagger \\
1,638 \ddagger\end{array}$ & $\begin{array}{l}75.0 \\
72.7 \\
72.8\end{array}$ & $\begin{array}{l}21.1 \\
18.8 \\
22.5\end{array}$ & $\begin{array}{l}\text { Duration } 8 \text { yrs. Refractory anemia. In- } \\
\text { adequate protein intake. Thyroid therapy } \\
\text { started } 3 \text { days after Ke. }\end{array}$ \\
\hline $3 \mathrm{~F}$ & 65 & Myxedema & $\begin{array}{l}1,679 \S \\
1,497\end{array}$ & $\begin{array}{l}62.4 \\
60.9\end{array}$ & $\begin{array}{l}27.9 \\
24.6\end{array}$ & $\begin{array}{l}\text { Classic symptoms and signs. Anemia for } 4 \\
\text { yrs. Hepatomegaly and ascites. Liver bi- } \\
\text { opsy showed fatty metamorphosis. No sup- } \\
\text { plementary K given. }\end{array}$ \\
\hline $4 \mathrm{M}$ & & Fever of unknown origin & $\begin{array}{l}1,814 \S \\
1,643\end{array}$ & $\begin{array}{l}47.7 \\
47.7\end{array}$ & $\begin{array}{l}38.0 \\
34.4\end{array}$ & $\begin{array}{l}\text { Duration of } 30 \text { days. No known weight loss. } \\
\text { Febrile during studies. }\end{array}$ \\
\hline $5 \mathrm{~F}$ & 46 & $\begin{array}{l}\text { Partial intestinal ob- } \\
\text { struction }\end{array}$ & $\begin{array}{l}1,235^{*} \\
1,344 \dagger\end{array}$ & $\begin{array}{l}45.5 \\
45.5\end{array}$ & $\begin{array}{l}27.1 \\
29.5\end{array}$ & $\begin{array}{l}\text { Due to postoperative adhesions. Symptoms } \\
\text { suggestive of peptic ulcer for } 8 \text { mos. } 10 \text { lbs. } \\
\text { loss in } 4 \text { mos. Diagnosis confirmed at } \\
\text { surgery. }\end{array}$ \\
\hline $6 \mathrm{~F}$ & 18 & $\begin{array}{l}\text { Rheumatic heart dis- } \\
\text { ease, active }\end{array}$ & $\begin{array}{r}1,014^{*} \\
941 \dagger\end{array}$ & $\begin{array}{l}45.9 \\
43.6\end{array}$ & $\begin{array}{l}22.1 \\
21.6\end{array}$ & $\begin{array}{l}\text { Recurrent rheumatic activity for } 7 \text { yrs. with } \\
\text { valvular heart disease, low grade fever and } \\
\text { elevated sedimentation rate. }\end{array}$ \\
\hline Group 4B & & & & & & \\
\hline $1 \mathrm{M}$ & 55 & Cirrhosis & $\begin{array}{l}2,084^{*} \\
2,550 \dagger \\
2,423 \ddagger\end{array}$ & $\begin{array}{l}75.5 \\
68.6 \\
68.2\end{array}$ & $\begin{array}{l}27.7 \\
37.2 \\
35.5\end{array}$ & $\begin{array}{l}\text { History of alcoholism for } 30 \text { yrs. with in- } \\
\text { adequate diet. Ankle edema, ascites for } 9 \\
\text { wks. before Ke.* }\end{array}$ \\
\hline $2 \mathrm{M}$ & 39 & Rheumatic heart disease & $\begin{array}{l}1,980^{*} \\
2,063 \dagger \\
2,315 \ddagger\end{array}$ & $\begin{array}{l}62.8 \\
58.9 \\
59.4\end{array}$ & $\begin{array}{l}31.5 \\
35.0 \\
39.0\end{array}$ & $\begin{array}{l}\text { Mitral stenosis and insufficiency, aortic insuf- } \\
\text { ficiency. Duration at least } 10 \text { yrs. On } \\
\text { digitalis. }\end{array}$ \\
\hline $3 \mathrm{~F}$ & 24 & Multiple sclerosis & $\begin{array}{l}1,380^{*} \\
1,657 \dagger\end{array}$ & $\begin{array}{l}49.5 \\
49.5\end{array}$ & $\begin{array}{l}27.9 \\
33.5\end{array}$ & Duration 3 yrs. Studied during a relapse. \\
\hline $4 \mathrm{M}$ & 41 & $\begin{array}{l}\text { Psychoneurosis, ade- } \\
\text { noma of thyroid }\end{array}$ & $\begin{array}{l}2,764^{*} \\
3,135 \dagger \\
2,967 \ddagger\end{array}$ & $\begin{array}{l}83.2 \\
83.2 \\
83.2\end{array}$ & $\begin{array}{l}33.2 \\
37.7 \\
35.7\end{array}$ & Vague complaints. Weakness prominent. \\
\hline $5 \mathrm{M}$ & & $\begin{array}{l}\text { Chronic glomerulone- } \\
\text { phritis }\end{array}$ & $\begin{array}{l}2,555^{*} \\
3,263 \dagger \\
2,505 \ddagger\end{array}$ & $\begin{array}{l}79.5 \\
77.3 \\
74.1\end{array}$ & $\begin{array}{l}32.1 \\
42.2 \\
33.8\end{array}$ & $\begin{array}{l}\text { Duration symptoms } 11 \text { yrs. Hypertension } 3 \\
\text { yrs. Worse past } 3 \text { mos. PSP }=9 \% \text { in } 1 \mathrm{hr} \text {. } \\
\text { NPN }=87 \mathrm{mg} . \% \text { with Ke. NPN increased } \\
\text { to } 154 \mathrm{mg} . \% \text { on day of Ke. } \neq\end{array}$ \\
\hline $6 \mathrm{M}$ & & Diabetes mellitus & $\begin{array}{l}1,553^{*} \\
1,873 \ddagger \\
1,463 \ddagger\end{array}$ & $\begin{array}{l}52.5 \\
48.3 \\
47.5\end{array}$ & $\begin{array}{l}29.6 \\
38.8 \\
30.8\end{array}$ & $\begin{array}{l}\text { Duration of diabetes } 5 \text { yrs. with poor regula- } \\
\text { tion. Peptic ulcer symptoms for } 2 \text { mos. } \\
\text { Developed pyloric obstruction and was placed } \\
\text { on nasogastric suction between Ke } \dagger \text { and Ke. } \\
\text { Metabolic alkalosis with Ke. } \ddagger\end{array}$ \\
\hline Group $4 \mathrm{~F}$ & 36 & $\begin{array}{c}\text { Chronic pulmonary fi- } \\
\text { brosis }\end{array}$ & $\begin{array}{l}2,928 * \\
2,157 \dagger \\
2,425 \ddagger\end{array}$ & $\begin{array}{l}52.6 \\
52.6 \\
52.6\end{array}$ & $\begin{array}{l}55.7 \\
41.0 \\
46.1\end{array}$ & $\begin{array}{l}\text { Chronic bronchitis progressive for } 13 \text { yrs., with } \\
\text { fibrosis. Prone to hyperventilate. } 36 \mathrm{lbs} \text {. } \\
\text { weight loss in } 2 \text { yrs. }\end{array}$ \\
\hline $2 \mathrm{M}$ & 52 & $\begin{array}{l}\text { Pneumoconiosis, gastric } \\
\text { ulcer }\end{array}$ & $\begin{array}{l}3,472^{*} \\
2,657 \dagger \\
1,776 t\end{array}$ & $\begin{array}{l}\mathbf{5 7 . 7} \\
\mathbf{5 7 . 7} \\
\mathbf{5 7 . 7}\end{array}$ & $\begin{array}{l}60.2 \\
46.0 \\
30.1\end{array}$ & $\begin{array}{l}\text { Progressive cough and dyspnea for } 16 \text { yrs. with } \\
\text { roentgenologic changes. Diagnosis of ulcer } \\
\text { established while hospitalized. }\end{array}$ \\
\hline $3 \mathrm{M}$ & 41 & Ulcerative colitis & $\begin{array}{l}3,113^{*} \\
2,319 \dagger \\
1,916 \ddagger\end{array}$ & $\begin{array}{l}68.2 \\
68.8 \\
66.8\end{array}$ & $\begin{array}{l}45.6 \\
33.8 \\
28.7\end{array}$ & $\begin{array}{l}\text { Duration } 1 \text { mo. } 14 \text { stools daily. } 40 \text { lbs. } \\
\text { weight loss in } 6 \text { mos. Asymptomatic peptic } \\
\text { ulcer. With } \mathrm{Ke}^{*} \text {, NPN }=76 \mathrm{mg} . \% \text {, with } \\
\text { Ket, NPN }=43 \mathrm{mg} . \% \text {. }\end{array}$ \\
\hline
\end{tabular}

Ke determinations were made at weekly intervals unless otherwise indicated.

* Ke before the administration of $\mathrm{KCl}$.

$\dagger \mathrm{Ke}$ after one week of $\mathrm{KCl}, 3 \mathrm{gm}$. daily.

$\ddagger \mathrm{Ke}$ one week after discontinuation of $\mathrm{KCl}$.

$\S$ Not given supplementary $\mathrm{KCl}$. 
heart disease, malnutrition, carcinoma, arteriosclerotic heart disease, thyrotoxicosis, and cirrhosis.

\section{Group 3 (Table III)}

The range of $\mathrm{Ke} / \mathrm{Wt}$. in 20 normal young adult females was previously found to be 25.1 to 35.9 meq. $/ \mathrm{Kg}$. Of the 22 individuals in group 2 , seven subjects had values higher than those found in the normal group. Four of the 22 had values below $25.1 \mathrm{meq} . / \mathrm{Kg}$. The diagnoses in these four subjects were, respectively: diabetes mellitus, psychoneurosis, obesity and anemia, and exogenous obesity with hypertensive cardiovascular disease.

\section{Group 4 (Table IV)}

The results of the study in this group have been divided into three categories on the basis of the type of response to supplementary potassium chloride. Group 4A consists of six individuals who did not show what was considered to be a significant change ( \pm 250 meq.) in $\mathrm{Ke}$ when placed on supplementary oral potassium chloride. Group 4B consists of six individuals who showed an increase in $\mathrm{Ke}$ at the second or third determination, or with both measurements. Group $4 \mathrm{C}$ consists of three individuals in whom the $\mathrm{Ke}$ value decreased during the administration of potassium supplements.

Group $4 A$. No change in the Ke occurred following the administration of potassium supplements in subjects with metastatic carcinoma, myxedema, fever of unknown origin, partial intestinal obstruction, and rheumatic heart disease.

Group $4 B$. The supplementary feeding of potassium chloride produced an increase in $\mathrm{Ke}$ of more than 250 meq. in the following disease states : cirrhosis, rheumatic heart disease, psychoneurosis, multiple sclerosis, chronic glomerulonephritis, and poorly regulated diabetes mellitus.

Group 4C. In these three instances, $\mathrm{Ke} \mathrm{de}-$ creased during the administration of supplementary potassium: chronic pulmonary fibrosis, pneumoconiosis and gastric ulcer, and ulcerative colitis.

\section{COMMENT}

It has been recognized in the past that in a few disease states certain abnormalities in potassium metabolism, as evidenced by alterations in the external balance or concentration in the serum, are associated with clinical symptoms and signs. A body deficiency of potassium is known to exist in diabetic acidosis and coma (4), in certain gastrointestinal disorders (5), and in certain postoperative conditions (6). With severe renal damage, retention of potassium may occur. The external balance method, however, gives no direct evidence of any imbalance in the distribution of potassium between the intra- and extracellular fluid compartments, although such an imbalance has been suspected. The difficulty of confirming clinically the suspicion of an intracellular potassium deficiency has been recognized previously. The finding of a low serum potassium concentration is highly suggestive of the presence of body deficiency. However, it is important to realize that a total body deficiency may exist in the presence of a normal serum concentration. All of the serum potassium determinations done on the patients in the present study were within the range of 3.5 to 5.0 meq. $/ \mathrm{Kg}$.

The availability of radioactive isotopes has provided a method for measuring directly the body content of potassium. Since more than $95 \%$ of the body's store of potassium is normally situated within cells, such a measurement of the total body content of potassium should serve as a relatively reliable index to the intracellular store of this cation.

Previous determinations of the exchangeable potassium content in normal male and female subjects have established presumably normal ranges $(1,2)$. Although it is recognized that such studies to date have been limited to fairly homogeneous groups of young subjects, these values are the only ones available at present for comparison with $\mathrm{Ke}$ values obtained in disease states.

The finding, in some diseased males and females, of $\mathrm{Ke} / \mathrm{Wt}$. values higher than those obtained in normal subjects suggests that the former group was more muscular than normal subjects. Such a finding suggests that the loss of body fat may elevate the relative fraction of lean tissue. Except in the case of the individual with chronic glomerulonephritis, it is difficult to explain the higher values for $\mathrm{Ke} / \mathrm{Wt}$. on the basis of a pathologic state. In severe renal failure, retention of potassium may occur when the intake exceeds the excretory capacity of the kidneys.

The results of the present study seem to indicate that several factors may produce a low $\mathrm{Ke} /$ Wt. value : 
1. A decrease in body mass secondary to (a) a decrease in food intake resulting from anorexia, (b) an increase in the catabolic processes, or (c) both.

The loss in total tissue mass, in such an event, would parallel the loss of body weight. The concentration of potassium in the remaining tissues, however, would be unchanged, so that no body deficiency of potassium would result. This factor is probably the one principally responsible for the low $\mathrm{Ke} / \mathrm{Wt}$. values in the individuals with malnutrition, malignancy, thyrotoxicosis, and chronic infectious diseases or febrile episodes. It has been shown experimentally that during starvation normal rabbits lose body potassium progressively with loss of body weight, but that the concentration of this cation in the remaining tissues is unaltered (7).

2. An increase in the relative fat content of the body, such as occurs with exogenous obesity (Table III, Cases 19 and 22).

3. Expansion of the extracellular fluid compartment as a result of edema or the deposition of myxedema fluid.

Should the low values for $\mathrm{Ke} / \mathrm{Wt}$. be due to any or all of the above causes, no decrease in the intracellular concentration of potassium occurs; therefore, no increase in the potassium content of the body would be expected when supplementary potassium is administered. Such was the result obtained in the cases of carcinoma, myxedema, chronic infectious disease, fever of unknown origin, and untreated rheumatic heart disease.

4. Excessive loss of potassium from the body, usually by the gastrointestinal tract, as the result of vomiting, diarrhea, or intestinal suction.

The potassium concentration of the intestinal secretions is several times higher than that of serum. With loss of intestinal contents, a decrease in the intracellular concentration of potassium results. Such a deficit might be corrected simply by supplying potassium orally or parenterally.

5. A cellular metabolic abnormality, which may lead to an intracellular deficiency.

A low value for $\mathrm{Ke} / \mathrm{Wt}$. may lead one to suspect such an abnormality. An increase in this value following the administration of supplementary potassium, particularly if such an increase is maintained after the supplement is discontinued, is certainly suggestive of a prior deficiency state. Such an intracellular potassium deficiency appears to be present in poorly regulated diabetes mellitus, and has already been shown to exist in diabetic acidosis and coma. A potassium deficit might be anticipated in cirrhotic patients because of the impairment in carbohydrate metabolism.

In the patients with psychoneurosis and multiple sclerosis, the increase in the $\mathrm{Ke}$ values following the administration of potassium supplement suggests the existence of an occult potassium deficiency in these states. Further studies are required to confirm these findings and to explain the mechanism.

\section{SUM MARY}

The use of a radioactive isotope of potassium, $\mathrm{K}^{42}$, has made possible the direct measurement of the exchangeable potassium content of the body. Such a determination was performed in 69 hospitalized subjects with various diseases. With few exceptions, the values obtained were either within the range found in normal young subjects, or lower. Although some of these low values could be attributed to the loss of body tissue resulting from chronic illness, the low values in certain disease states suggested the presence of a body deficiency of the potassium ion.

\section{REFERENCES}

1. Corsa, L., Olney, J. M., Jr., Steenburg, R. W., Ball, M. R., and Moore, F. D., The measurement of exchangeable potassium in man by isotope dilution. J. Clin. Invest., 1950, 29, 1280.

2. Aikawa, J. K., Harrell, G. T., and Eisenberg, B., The exchangeable potassium content of normal women. J. Clin. Invest., 1952, 31, 367.

3. Mosher, R. E., Boyle, A. J., Bird, E. J., Jacobson, S. D., Batchelor, T. M., Iseri, L. J., and Myers, G. B., The use of flame photometry for the quantitative determination of sodium and potassium in plasma and urine. Am. J. Clin. Path., 1949, 19, 461.

4. Danowski, T. S., Peters, J. H., Rathbun, J. C., Quashnock, J. M., and Greenman, L., Studies in diabetic acidosis and coma, with particular emphasis on the retention of administered potassium. J. Clin. Invest., 1949, 28, 1.

5. Elkinton, J. R., Squires, R. D., and Crosley, A. P., Jr., Intracellular cation exchanges in metabolic alkalosis. J. Clin. Invest., 1951, 30, 369.

6. Randall, H. T., Habif, D. V., Lockwood, J. S., and Werner, S. C., Potassium deficiency in surgical patients. Surgery, 1949, 26, 341.

7. Aikawa, J. K., Effect of starvation on exchangeable potassium and tissue $\mathrm{K}^{42}$ content in rabbits. Proc. Soc. Exper. Biol. \& Med., 1951, 78, 524. 